BOTANICA

ISSN 2538-8657

2020, 26(2): 197-200

\title{
A CHECKLIST OF LATVIAN MYXOMYCETES
}

\section{Gražina Adamonytė}

Žaliujų Ežerų Str. 3-88, 08408 Vilnius, Lithuania

E-mail: grazina_@yahoo.com

\begin{abstract}
Adamonytė G., 2020: A checklist of Latvian myxomycetes. - Botanica, 26(2): 197-200.

The paper presents a list of myxomycete species recorded so far in Latvia. The list is based on literature records and includes 108 species.
\end{abstract}

Keywords: Latvia, myxomycetes, slime molds.

\section{INTRODUCTION}

Myxomycetes, or slime molds, are a group of unicellular heterotrophic eukaryotes usually inhabiting terrestrial ecosystems and feeding on bacteria and other microorganisms in decaying plant material. Their life cycle includes several developmental stages: trophic stages (myxamoebae, swarm cells, and plasmodia), resting or dormant stages (spores, microcysts and sclerotia), and developing fruiting bodies with internally formed spores (EVERHART \& KeLler, 2008). Approximately 1000 morphological species are known throughout the world, but the level of knowledge about the diversity of myxomycete species differs among regions and countries. Only a few publications devoted to this group of organisms in Latvia are available, the earliest of these, ROTHERT (1890), with the quite impressive list of 40 species collected near Rīga. Later, Bucholtz' (1908) paper followed, which was a compilation of myxomycete data presented by several authors from "Russland Ostseeprovinzen", and with Latvian records represented mostly by Rothert. Further on, KuPPFER (1931) listed 22 species of myxomycetes in his geobotanical study of the Moricsala Nature Reserve, in the northwestern part of Latvia. After a long gap in myxomycete investigations in Latvia, 40 species were recorded from Slītere National Park, Talsi distr. (RUSKule \&
VIMBA, 1987). A few papers exclusively dealing with Latvian myxomycetes appeared in the early 2000s (AdAmonyté \& Vimba, 2003; etc.). The present paper aims to summarize the so-far available records of myxomycetes from the territory of Latvia.

\section{MATERIALS AND METHODS}

The species list, including also the genus Ceratiomyxa J. Schröt. with externally formed spores, is based on published sources listed in References. The herbarium material was not revised. In the list, the currently accepted species name is followed by the name under which it was published for the first time (if different) and by the first published reference. The nomenclature was updated following LADO (20052020). The names of authors were abbreviated according to BRUMmit \& Powell (1992).

\section{LIST OF MYXOMYCETE SPECIES}

Amaurochaete atra (Alb. et Schwein.) Rostaf. ROTHERT, 1890.

Arcyodes incarnata (Alb. et Schwein.) O.F.Cook as Lachnobolus incarnatus Alb. et Schw. RusKule \& VimBA, 1987.

Arcyria affinis Rostaf. - AdAmonytė \& VIMBA, 2005. 
Arcyria cinerea (Bull.) Pers. - Rothert, 1890.

Arcyria denudata (L.) Wettst. as Arcyria punicea Pers. - Rothert, 1890.

Arcyria glauca Lister - Ruskule \& VimBA, 1987.

Arcyria incarnata (Pers. ex J.F. Gmel.) Pers. -

ROTHERT, 1890.

Arcyria insignis Kalchbr. \& Cooke - Ruskule \& VIMBA, 1987.

Arcyria minuta Buchet - AdAMONYTE, 2006.

Arcyria obvelata (Oeder) Onsberg as Arcyria nutans

Greville - ROTHERT, 1890.

Arcyria oerstedii Rostaf. - Vimba \& AdAmonytE, 2003.

Arcyria pomiformis (Leers) Rostaf. - ROTHERT, 1890.

Arcyria stipata (Schwein.) Lister - AdAMONYTE, 2006.

Badhamia macrocarpa (Ces.) Rostaf. - VIMBA \& AdAMONYTE், 2003.

Badhamia panicea (Fr.) Rostaf - AdAmONYTĖ, 2006.

Badhamia utricularis (Bull.) Berk. - ADAMONYTE \& VIMBA, 2005.

Barbeyella minutissima Meyl. - AdAMONYtĖ, 2006.

Calomyxa metallica (Berk.) Nieuwl. - AdAMONYTÉ, 2006.

Ceratiomyxa fruticulosa (O.F. Müll.) T. Macbr. as Ceratiomyxa mucida Schröter and Ceratiomyxa porioides Schröter - ROTHERT, 1890; as Ceratiomyxa pyxidata (Alb. et Schw.) Schrt. KUPFFER, 1931.

Clastoderma debaryanum A. Blytt - AdAMONYTE, 2006.

Collaria arcyrionema (Rostaf.) Nann.-Bremek. ex Lado as Lamproderma arcyrionema Rostaf. ROTHERT, 1890.

Colloderma oculatum (C. Lippert) G. Lister ADAMONYTE், 2006.

Comatricha elegans (Racib.) G. Lister as Collaria elegans (Racib.) Dhillon et Nann.-Bremek. ex Ing - Vimba \& Adamonyté, 2003.

Craterium leucocephalum (Pers. ex J.F. Gmel.) Ditmar - RotherT, 1890.

Cribraria argillacea (Pers. ex J.F. Gmel.) Pers. ROTHERT, 1890.

Cribraria aurantiaca Schrad. - Rothert, 1890.

Cribraria cancellata (Batsch) Nann.-Bremek. as Dictydium cancellatum Macbr. - ROTHERT, 1890.
Cribraria ferruginea Meyl. - Ruskule \& VimBA, 1987.

Cribraria macrocarpa Schrad. - RotheRT, 1890.

Cribraria purpurea Schrad. - Ruskule \& VimBA, 1987.

Cribraria rufa (Roth.) Rostaf. - RotheRt, 1890.

Cribraria violacea Rex - AdAMONYTE, 2006.

Cribraria vulgaris Schrad. - AdAmONytė \& Vimba, 2005.

Dianema corticatum Lister - ADAMONYTE், 2006.

Diderma cinereum Morgan - Ruskule \& VIMBA, 1987.

Diderma floriforme (Bull.) Pers. - AdamonytĖ \& VIMBA, 2005.

Diderma radiatum (L.) Morgan as Chondrioderma radiatum Rostafinski - RoTHERT, 1890.

Diderma spumarioides (Fr. et Palmquist) Fr. as Chondrioderma spumarioides Rostafinski RotHERT, 1890.

Didymium bahiense Gottsb. - AdAmonytė \& Vimba, 2003.

Didymium difforme (Pers.) Gray. - AdAmonyte \& VIMBA, 2003.

Didymium iridis (Ditmar) Fr. - VIMBA \& AdAMONYTE, 2003.

Didymium melanospermum (Pers.) T. Macbr. as Didymium farinaceum Schrad. - RotherT, 1890.

Didymium minus (Lister) Morgan - VIMBA \& ADAMONYTÉ, 2003.

Didymium nigripes (Link) Fr. - ROTHERT, 1890.

Didymium squamulosum (Alb. et Schwein.) Fr. et Palmquist - RusKULE \& VIMBA, 1987.

Echinostelium apitectum K.D. Whitney-ADAMONYTE, 2006.

Echinostelium brooksii K.D. Whitney - ADAMONYTE, 2006.

Echinostelium minutum de Bary - AdAMONYTE, 2006.

Enerthenema papillatum (Pers.) Rostaf. - ROTHERT, 1890.

Fuligo leviderma H. Neubert, Nowotny et K. Baumann - ADAMONYTÉ, 2006.

Fuligo muscorum Alb. et Schwein. - Ruskule \& VIMBA, 1987.

Fuligo septica (L.) F.H. Wigg. as Fuligo septica Gmelin. - Rothert, 1890, as Fuligo candida Pers. - Vimba \& AdAmonyté, 2003. 
Hemitrichia calyculata (Speg.) M.L. Farr AdAMONYTÉ, 2006.

Hemitrichia clavata (Pers.) Rostaf. - ROTHERT, 1890.

Hemitrichia leiotricha (Lister) G. Lister - RUSKULE \& VIMBA, 1987.

Hemitrichia serpula (Scop.) Rostaf. ex Lister - RusKULE \& VIMBA, 1987.

Lamproderma arcyrioides (Sommerf.) Rostaf. Vimba \& AdAMONYTE, 2003.

Lamproderma columbinum (Pers.) Rostaf. ADAMONYTÉ, 2006.

Leocarpus fragilis (Dicks.) Rostaf. as Leocarpus fragilis Rostafinski - ROTHERT, 1890.

Licea kleistobolus G.W. Martin - AdAmonyté, 2006.

Licea minima Fr. - AdAMONYTÉ, 2006.

Licea operculata (Wingate) G.W. Martin - AdAmoNYTE், 2006.

Licea parasitica (Zukal) G.W. Martin - AdAMONYTE, 2006.

Licea pusilla Schrad. - AdAmONYté, 2006.

Licea pygmaea (Meyl.) Ing - ADAMONYTE், 2006.

Licea variabilis Schrad. as Licea flexuosa Pers. ROTHERT, 1890.

Lindbladia tubulina Fr. as Lindbladia effusa (Ehrenb.) Rostaf. - Rothert, 1890.

Lycogala epidendrum (L.) Fr. - Rothert, 1890.

Lycogala exiguum Morgan - Ruskule \& VIMBA, 1987.

Macbrideola cornea (G. Lister et Cran) Alexop. ADAMONYTÉ, 2006.

Metatrichia floriformis (Schwein.) Nann.-Bremek. VimBa \& AdAMONYTÉ, 2003.

Metatrichia vesparia (Batsch) Nann.-Bremek. ex G.W. Martin et Alexop. as Hemitrichia vesparium Macbr. - JACZEWSKI, 1907.

Mucilago crustacea P. Micheli ex F.H. Wigg. as Spumaria alba DC - RotheRT, 1890.

Paradiacheopsis fimbriata (G. Lister et Cran) Hertel ex Nann.-Bremek. - AdAMONYTÉ, 2006.

Paradiacheopsis solitaria (Nann.-Bremek.) Nann.Bremek. - AdAMONYTÉ, 2006.

Perichaena chrysosperma (Curr.) Lister AdAMONYTĖ, 2006.

Perichaena corticalis (Batsch) Rostaf. - ADAMONYTĖ \& VIMBA, 2005.

Perichaena pedata (Lister et G. Lister) G. Lister ex E. Jahn - AdAMONYTÉ, 2006.
Physarum album (Bull.) Cheval. as Physarum nutans Pers. - Rothert, 1890.

Physarum altaicum Lavrov - RUSKULE \& VIMBA, 1987.

Physarum cinereum (Batsch) Pers. - KuPfER, 1931.

Physarum citrinum Schumach. - ADAMONYTE \& VIMBA, 2003.

Physarum compressum Alb. et Schwein. - Ruskule \& VIMBA, 1987.

Physarum contextum (Pers.) Pers. - Rothert, 1890.

Physarum flavicomum Berk. - AdAMONYTE \& VimBA, 2003.

Physarum globuliferum (Bull.) Pers. - ROTHERT, 1890.

Physarum leucopus Link - Ruskule \& Vimba, 1987.

Physarum psittacinum Ditmar - KUPFER, 1931.

Physarum robustum (Lister) Nann.-Bremek. - VIMBA \& AdAMONYTE், 2003.

Physarum virescens Ditmar - Rothert, 1890.

Physarum viride (Bull.) Pers. as Physarum viride Pers. - Rothert, 1890.

Reticularia lycoperdon Bull. - ROTHERT, 1890.

Reticularia jurana Meyl. as Enteridium splendens var. juranum (Meyl.) Härk. - ADAMONYTĖ \& VIMBA, 2003.

Stemonitis axifera (Bull.) T. Macbr. as Stemonitis ferruginea Ehrenb. - Rothert, 1890, as Stemonitis smithii T. Macbr. - RUSKULE \& VIMBA, 1987.

Stemonitis fusca Roth. - Rothert, 1890.

Stemonitopsis hyperopta (Meyl.) Nann.-Bremek. Adamonyté \& Vimba, 2003.

Stemonitopsis typhina (F. H. Wigg.) Nann.-Bremek. as Comatricha typhina Rostaf. - ROTHERT, 1890.

Symphytocarpus amaurochaetoides Nann.-Bremek.Adamonyté \& Vimba, 2003.

Symphytocarpus flaccidus (Lister) Ing et Nann.Bremek. - AdAMOnYté \& VimBa, 2003.

Thecotubifera dictyoderma (Nann.-Bremek. \& Loer.) Leontyev, Schnittler, S.L.Stephenson \& Novozh. as Tubifera dictyoderma Nann.Bremek. et Loer. - AdAMONYTÉ, 2006.

Trichia botrytis (J.F. Gmel.) Pers. - KupfFer, 1931.

Trichia contorta (Ditmar) Rostaf. - RUSKULE \& VIMBA, 1987.

Trichia decipiens (Pers.) T. Macbr. - ROTHERT, 1890. 
Trichia favoginea (Batsch) Pers. as Trichia jackii Rostaf. - KUPFER, 1931.

Trichia persimilis P. Karst. - RotheRT, 1890.

Trichia scabra Rostaf. - ROTHERT, 1890.

Trichia varia (Pers. ex J.F. Gmel.) Pers. - RotherT, 1890.

Tubifera ferruginosa (Batsch) J.F. Gmel. as Tubulina cylindrica (Bull.) DC - ROTHERT, 1890.

A total of 108 species were included in the list. Compared to the countries similar in size and climate to Latvia, e. g. Denmark, where the list consists of at least 221 species (GøтZsCHE, 2005-2020) or Estonia, where 150 species of myxomycetes have recently been listed (YATSIUK et al., 2020), it is evident that greater scope of research will result in a considerable number of myxomycete species new to Latvia.

\section{ACKNOWLEDGEMENTS}

Thanks are extended to Prof. Uno H. Eliasson for his encouragement and valuable suggestions during manuscript preparation. This paper is dedicated to an outstanding Latvian mycologist Edgars Vīmba on the occasion of his 90th anniversary.

\section{REFERENCES}

AdAmonytė G., 2006: New findings of myxomycetes in Latvia. - Botanica Lithuanica, 12(1): 57-64.

Adamonytė G., Vimba E., 2003: Unpublished myxomycete collection of K.R. Kupffer. - Folia Cryptogamica Estonica, Fasc. 40: 1-6.

Adamonyté G., Vimba E., 2005: Notes on a collection of myxomycetes by K. Starcs in the herbarium of the University of Latvia. - XVI Symposium of My- cologists and Lichenologists of the Baltic States, 21-25 September 2005, Cesis, Latvia: 43-47.

Brummitt R.K., Powell C.E. (eds), 1992: Authors of plant names. - Kew.

Bucholtz F., 1908: Verzeichnis der bisher für die Ostseeprovinzen Russlands bekannt gewordenen Myxogasteres. - Korrespondenzblatt des Naturforscher-Vereins zu Riga, 51: 93-108.

Everhart S.E., Keller H.W., 2008: Life history strategies of corticolous myxomycetes: the life cycle, plasmodial types, fruiting bodies, and taxonomic orders. - Fungal Diversity, 29: 1-16.

Gøtzsche H.F., 2005-2020: Checklist of Danish Myxomycetes. - http://www.myx.dk/dk/dkchklst.html [Accessed 2020 May].

JACZEWSKY A.A., 1907: Mikologičeskaja flora evropejskoj i aziatskoj Rossii (Slizeviki), 2. - Moskva.

KUPFFer K.R., 1931: Die Naturschonstätte Moritzholm. - Arbeiten des Naturforscher Vereins zu Riga. Neue Folge, XIX: 53-101.

LADO C., 2005-2020: An online nomenclatural information system of Eumycetozoa. - http://www.nomen.eumycetozoa.com (consulted 2020 May).

Rothert W., 1890: Die bei Riga gefundenen Myxomyceten. - Scripta botanica Horti Universitatis Imperialis Petropolitanae, III(fasc. 1): 1-13 (In Russian).

Ruskule S., Vimba E., 1987: Investigations of myxomycetes of Slītere National Park. Mežsaimniecība un Mežrūpniecība, 2: 41-42 (in Latvian).

Vimba E., Adamonytė G., 2003: Additional data on Latvian myxomycetes. - Folia Cryptogamica Estonica, 40: 57-61.

Yatsiuk I., Adamonytė G., Kastanje V., 2020: A comprehensive checklist of Estonian myxomycetes. Karstenia, 58 (2): 241-249.

\section{LATVIJOS GLEIVŪNŲ SĄRAŠAS}

\section{Gražina ADAMONYTE்}

\section{Santrauka}

Straipsnyje pateikiamas visų Latvijoje iki šiol užregistruotų gleivūnų rūšių sąrašas, sudarytas re- miantis literatūros šaltiniais. Sąrašą sudaro 108 gleivūnų rūšys. 